\title{
THE SUBNORMAL STRUCTURE OF SOME CLASSES OF SOLUBLE GROUPS
}

\author{
D. McDOUGALL \\ (Received 15 December 1969) \\ Communicated by G. E. Wall
}

\section{Introduction}

Finite groups in which normality is transitive have been studied by Best and Taussky, [1], Gaschütz, [3], and Zacher [16]. Infinite soluble groups in which normality is transitive have been studied by Robinson in [9]. A subgroup $H$ of a group $G$ is subnormal in $G$ if $H$ can be connected to $G$ by a chain of $r$ subgroups, in which each is normal in its successor, where $r$ is a non-negative integer. The least such $r$ is called the subnormal index of $H$ in $G$ (or the defect of $H$ in $G$ ). Then groups in which normality is transitive are precisely those in which every subnormal subgroup has subnormal index at most one. Thus the structure of soluble groups in which every subnormal subgroup has subnormal index at most $n$ (such a group is said to have bounded subnormal indices) has been dealt with by Robinson in [9] for the case where $n$ is one. However Theorem $D$ of [12] states that a soluble group of derived length $n$ can be embedded in a soluble group in which the subnormal indices are at most $n$. Therefore we must impose further conditions on the groups if we hope to obtain any worthwhile results for the above problem with $n$ greater than one.

In this paper we consider the structure of soluble $p$-groups which have a bound on their subnormal indices. The investigation involves a class of periodic soluble groups which, for want of a better terminology, we call $\mathbf{p} \mathscr{Q}$ groups. This class of groups does not appear to have been studied elsewhere and so the first part of this paper is concerned with giving some of its interesting properties. An idea of the complexity of this class can be obtained from Theorem 2.1 which states that every soluble group of finite exponent can be embedded in a $\mathbf{P} 2$-group of the same derived length.

\section{The classes $\mathbf{P} \mathscr{Q}, \dot{P} \mathscr{Q}, \dot{P}_{\mathrm{N}} \mathscr{Q}$}

Definition. A group $G$ is radicable if every element of $G$ has an $m$-th root for every positive integer $m$. Let $\mathscr{Q}$ denote the class of periodic radicable abelian 
groups. Then, using the usual closure operator notation (see for instance [13]), $G$ is a $\mathbf{P} \mathscr{Q}$ group if and only if there is a series

$$
1=G_{0} \leqq G_{1} \leqq G_{2} \leqq \cdots \leqq G_{n-1} \leqq G_{n}=G
$$

in which each $G_{i}$ is normal in $G_{i+1}$ and $G_{i+1} / G_{i}$ is a $\mathscr{Q}$ group. There are two obvious ways of forming generalizations of this class.

Definition. A group $G$ is a $\mathbf{P} \mathscr{Q}$ group if and only if there is a transfinite ascending series $\left\{G_{\alpha}\right\}_{\alpha \leqq p}$ in $G$ with $G=G_{\rho}$ and each $G_{\alpha+1} / G_{\alpha}$ a $\mathscr{Q}$ group.

Definition. A group $G$ is a $\dot{\mathbf{P}}_{\mathrm{N}} \mathscr{Q}$ group if and only if there is a transfinite ascending series $\left\{G_{\alpha}\right\}_{\alpha \leqq \rho}$ in $G$ with $G=G_{\rho}$, each $G_{\alpha}$ normal in $G$, and each $G_{\alpha+1} / G_{\alpha}$ lying in 2 . ( $\hat{\mathbf{P}}_{\mathbf{N}}$ is not a closure operation).

Let $G^{m}$ denote the subgroup of $G$ generated by the $m$-th powers of the elements of $G$. Then we can generalise the concept of radicability as follows:

Definition. A group $G$ is quasi-radicable if $G=G^{m}$ for all positive integers $m$.

It is easy to see that the groups in $\mathbf{P} \mathscr{Q}, \dot{P} \mathscr{Q}$ and $\dot{P}_{\mathrm{N}} \mathscr{Q}$ are all quasi-radicable, by an induction argument (transfinite for the last two classes).

The following theorem proves the existence of sufficiently many $\mathbf{P} \mathscr{Q}$ groups to justify further investigation, and indicates that our previous definitions give rise to non-trivial classes of groups.

THEOREM 2.1. Every soluble group of finite exponent can be embedded in a $\mathrm{P} \mathscr{Q}$ group of the same derived length.

Proof. Suppose $G$ is a soluble group of finite exponent and has derived length $n$. We may assume $n$ is greater than one since the result is well-known for abelian groups (see for example Kuroš, [7]). We use induction on $n$. Let $A=G^{(n-1)}$ (where $G^{(1)}=G^{\prime}$, the derived group of $G$ and $\left.G^{(n)}=\left(G^{(n-1)}\right)^{\prime}\right)$. Then $A$ is an abelian group of finite exponent and $G / A$ is a soluble group of finite exponent with derived length $n-1$. By the well-known result of Krasner and Kaloujnine [6] we can embed $G$ in $A W r G / A$, the unrestricted standard wreath product of $A$ and $G / A$. If $H$ is the base group of $A W r G / A$ then $H$ is periodic since $A$ has finite exponent. But $A$ can be embedded in $A^{*} \in \mathscr{Q}$ and by the induction hypothesis $G / A$ can be embedded in $B^{*}$, a $\mathbf{P} \mathscr{Q}$ group of derived length $n-1$. Then $G$ can be embedded in $A W r G / A$ which in turn can be embedded in $A^{*} W r B^{*}=G^{*}$. If $H^{*}$ is the base group of $G^{*}$ then $H$ is embedded in tor $H^{*}$, the torsion subgroup of $H^{*}$. Thus $A W r G / A$ is embedded in (tor $H^{*}$ ) $B^{*}$. Therefore $G$ can be embedded in (tor $\left.H^{*}\right) B^{*}$ which is a $\mathrm{P} \mathscr{Q}$ group of derived length $n$, as required.

\section{Preliminary results and definitions}

Definition. Let $H$ be a subgroup of a group $G$. Then $H$ is subnormal in $G$ if and only if for some integer $r \geqq 0$ 


$$
H=H_{r} \triangleleft H_{r-1} \triangleleft H_{r-2} \cdots \triangleleft H_{0}=G
$$

(where $H \triangleleft G$ means that $H$ is a normal, not necessarily proper, subgroup of $G)$. We usually write $H \triangleleft^{r} G$.

Definition. For any subgroup $H$ of a group $G$ we define the standard series of $H$ in $G$ as follows:

$H^{G, 0}=G$; if $\alpha$ is not a limit ordinal let $H^{G, \alpha}$ be the normal closure of $H$ in $H^{G, \alpha-1}$; if $\alpha$ is a limit ordinal let $H^{G, \alpha}=\bigcap_{\beta<\alpha} H^{G, \beta}$.

It is easy to show that $H \triangleleft^{r} G$ if and only if $H=H^{G, r}$, and it is well-known that $H^{G, r}=H \gamma G H^{r}$ (where $\gamma G H^{r}$ denotes $\left.[G, H, \cdots, H]\right)$.

Definition. The least $r \geqq 0$ such that $H=H^{G, r}$ is the index of subnormality of $H$ in $G$, and is denoted by $s(G: H)$.

The standard series provides a simple criterion for a group to have a bound on its subnormal indices.

LEMMA 3.1. (Lemma 2 (i) of [10]). A group $G$ has a bound for its subnormal indices if and only if for each $H \leqq G, H^{G, n}=H^{G, n+1}=\cdots$, where $n$ is independent of $H$.

The next lemma is really the key to what follows.

Lemma 3.2. (2.2 of [11]). Let $A, H$ be subnormal subgroups of $G$. Suppose $A \in \mathscr{Q}$. If $H / H^{\prime}$ is periodic then $A$ normalises $H$. If in addition $H$ is nilpotent then $[H, A]=1$.

CoRollary 3.3. $\mathscr{Z}$ is $\mathrm{N}$-closed, that is if $G$ is generated by subnormal $\mathscr{Z}$ subgroups then $G$ is a $\mathscr{Z}$ group.

Definition. A subgroup $H$ of a group $G$ is ascendant in $G$ if there is an ascending (transfinite) chain of subgroups of $G$, each normal in its successor, connecting $H$ to $G$.

Lemma 3.4. (Corollary to lemma 4.3 of [11]). In any group an ascendant $C_{p} \infty$ subgroup is subnormal.

COROLlARY 3.5. In any group an ascendant $\mathscr{Q}$ subgroup is subnormal.

Proof. If $H$ is ascendant in $G$ and $H$ is a $\mathscr{Q}$ group then $H$ is a direct product of groups of type $C_{p} \infty$ for various primes. By 3.4 each of these $C_{p} \infty$ subgroups of $G$ is subnormal. Thus $H$ is contained in the subgroup generated by all the subnormal $\mathscr{Q}$ subgroups of $G$. But by 3.3 this last subgroup is a $\mathscr{Q}$ group and is clearly normal in $G$. Hence $H$ is subnormal in $G$.

\section{Properties of $P \mathscr{Q}$ groups}

Definition. For any group $G$ let $K_{1}(G)$ be the subgroup of $G$ generated by all the ascendant $\mathscr{Q}$ subgroups of $G$. Let $K_{0}(G)=1$; if $\alpha$ is a limit ordinal let 
$K_{\alpha}(G)=\bigcup_{\beta<\alpha} K_{\beta}(G)$; if $\alpha$ is not a limit ordinal let $K_{\alpha}(G) / K_{\alpha-1}(G)=K_{1}\left(G / K_{\alpha-1}\right.$ $(G)$ ). But by 3.3 and $3.4 K_{1}(G)$ is a 2 -group. Hence $\left\{K_{\alpha}(G)\right\}$ is an ascending series of characteristic subgroups of $G$ in which the factors are $\mathscr{2}$-groups. We call $\left\{K_{\alpha}(G)\right\}$ the upper-Q2-series of $G$. This terminology is justified during the proof of the next lemma.

Lemma 4.1. ' $\mathscr{P} \mathscr{Q}=\dot{\mathrm{P}}_{\mathrm{N}} 2$. Also $G \in \mathbf{P} \mathscr{Q}$ if and only if $G=K_{n}(G)$ for some positive integer $n$.

Proof. Clearly $\dot{P}_{2} \mathscr{Q} \geqq \dot{\mathbf{P}}_{\mathbf{N}} \mathscr{2}$. Suppose $G \in \dot{\mathbf{P}} \mathscr{2}$. Let $\left\{G_{\alpha}\right\}_{\alpha \leqq p}$ be an ascending 2-series in $G$ with $G=G_{p}$. We will show by transfinite induction that $G_{\alpha} \leqq K_{\alpha}(G)$ for all $\alpha \leqq \rho$. Note that this will justify our use of the word 'upper' in the terminology.

Suppose $G_{\beta} \leqq K_{\beta}(G)$ for all $\beta<\alpha$. Suppose $\alpha$ is a limit ordinal. Then $G_{\alpha}=\bigcup_{\beta<\alpha} G_{\beta} \leqq \bigcup_{\beta<\alpha} K_{\beta}(G)=K_{\alpha}(G)$. If $\alpha$ is not a limit ordinal then $G_{\alpha} K_{\alpha-1}(G) /$ $K_{\alpha-1}(G)$ is an ascendant subgroup of $G / K_{\alpha-1}(G)$. Also $G_{\alpha} K_{\alpha-1}(G) / K_{\alpha-1}(G)$ $\cong G_{\alpha} / G_{\alpha} \cap K_{\alpha-1}(G)$, which is a homomorphic image of $G_{\alpha} / G_{\alpha-1}$ since $K_{\alpha-1}(G)$ $\geqq G_{\alpha-1}$ by hypothesis.

Since $G_{\alpha} / G_{\alpha-1}$ is a 2 group it follows that $G_{\alpha} K_{\alpha-1}(G) / K_{\alpha-1}(G)$ also belongs to 2 . Hence by the definition of $K_{\alpha}(G)$ we have $G_{\alpha} K_{\alpha-1}(G) \leqq K_{\alpha}(G)$. Therefore $K_{\alpha}(G) \geqq G_{\alpha}$ for all $\alpha \leqq \rho$. Since $G_{\rho}=G$ this means that $K_{\rho}(G)=G$ and hence $G \in \dot{\mathrm{P}}_{\mathrm{N}} \mathscr{Q}$. Hence $\dot{\mathrm{P}}_{\mathrm{N}} \mathscr{Q} \geqq \dot{\mathrm{P}} \mathscr{Q}$, and so we have equality.

In particular if $\rho$ is finite so that $G \in \mathrm{P} \mathscr{Q}$, then $G=K_{n}(G)$ where $n=\rho$, an integer. Conversely if $G=K_{n}(G)$ then $G \in \mathrm{P} \mathscr{Q}$ and the proof is complete.

The class $\mathscr{Q}$ is not subgroup-closed, and so $\mathrm{P} \mathscr{Q}$ is not subgroup closed. But certain non-trivial subgroups of a $\mathbf{P} \mathscr{Q}$ group a $\mathbf{P} \mathscr{2}$ subgroup are $\mathbf{P} \mathscr{Q}$ groups, as the following lemma shows.

Lemma 4.2. If $G \in \dot{\mathrm{P}} \mathscr{Q}$ then $G^{\prime} \in \dot{\mathrm{P}} \mathscr{Q}$, and if $G \in \mathrm{P} \mathscr{Q}$ then $G^{\prime} \in \mathrm{P} \mathscr{Q}$.

Proof. Suppose firstly that $1 \leqq A \sqsupset G$, where $A$ and $G / A$ are both 2 groups. Let $g \in G$. Then for $a \in A$ the mapping $a \rightarrow[a, g]$ is a homomorphism. For if $a_{1}, a_{2} \in A, a_{1} a_{2} \rightarrow\left[a_{1} a_{2}, g\right]=\left[a_{1}, g\right]\left[a_{1}, g, a_{2}\right]\left[a_{2}, g\right]=\left[a_{1}, g\right]\left[a_{2}, g\right]$. Thus $[A, g]$ is a homomorphic image of $A$, and hence is a 2 -group. Therefore $[A, G]=\prod_{g \varepsilon G}[A, g]$ is a 2 group. Writing $G /[A, G]=H$ we have that $A /[A, G]$ is a central $\mathscr{Q}$ subgroup of $H$, with the factor group isomorphic to $G / A \in \mathscr{Q}$. As remarked in Section $2 H$ is quasi-radicable. But $H$ is a periodic nilpotent group, so by a result of Černikov, [2], $H$ is abelian. This means that $G^{\prime} \leqq[A, G] \leqq G^{\prime}$. Thus $G^{\prime}=[A, G] \in \mathscr{Q}$.

By $4.1 \dot{\mathbf{P}} \mathscr{Q}=\dot{\mathbf{P}}_{\mathrm{N}} \mathscr{Q}$, so we can assume $K_{\rho}(G)=G$. We show by transfinite induction that $K_{\alpha}(G)^{\prime} \in \dot{\mathbf{P}} \mathscr{Q}$ for all $\alpha$. Suppose $\alpha$ is not a limit ordinal. Consider $K_{\alpha-1}(G)^{\prime} \leqq K_{\alpha-1}(G) \leqq K_{\alpha}(G)$. Now $K_{\alpha}(G) / K_{\alpha-1}(G)$ and $K_{\alpha-1}(G) / K_{\alpha-1}(G)^{\prime}$ are both $\mathscr{Q}$ groups, so by the above argument applied to $K_{\alpha}(G) / K_{\alpha-1}(G)^{\prime}$ we have that $K_{\alpha}(G)^{\prime} / K_{\alpha-1}(G)^{\prime} \in \mathscr{Q}$. By hypothesis $K_{\alpha-1}(G)^{\prime} \in \dot{\mathbf{P}} \mathscr{Q}$, and so $K_{\alpha}(G)^{\prime} \in \dot{P}^{\prime} \mathscr{L}$. Suppose 
$\alpha$ is a limit ordinal. Then $K_{\alpha}(G)=\bigcup_{\beta<\alpha} K_{\beta}(G)$. By hypothesis each $K_{\beta}(G)^{\prime}$ is a $\mathbf{P} 2$ group. Hence $K_{\alpha}(G)^{\prime}=\bigcup_{\beta<\alpha} K_{\beta}(G)^{\prime} \in \mathrm{N}(\dot{P} \mathscr{Q})$. But since $\mathbf{P} \mathscr{Q}$ is $\mathbf{P}$ and quotientclosed it follows that $\mathbf{P} \mathscr{Q}$ is $\mathrm{N}$-closed, by a result of $\mathbf{P}$. Hall, [5] (p 537). Therefore $K_{\alpha}(G)^{\prime} \in \mathbf{P} \mathscr{Q}$ for all $\alpha \leqq \rho$. Thus $G^{\prime}=K_{\rho}(G) \in \hat{\mathbf{P}}^{\prime} \mathscr{Q}$.

In particular if $\rho$ is finite so that $\rho=n$, then clearly $G^{\prime}=K_{n}(G)^{\prime} \in \mathbf{P} \mathscr{Q}$.

The next lemma will be of use later.

Lemma 4.3. If $G$ is a soluble group of derived length $d$ then the length of the upper 2 series of $G$ is at most $d$.

Proof. Consider $K_{d+1}(G)$. This has derived length at most $d$ since it is a subgroup of $G$. But by continued application of 4.2 we have

$1 \leqq K_{d+1}(G)^{(d-1)} \leqq K_{d+1}(G)^{(d-2)} \leqq \cdots \leqq K_{d+1}(G)^{\prime} \leqq K_{d+1}(G)$, where each factor $\in$ 2. By our justification of the 'upper' in our terminology in 4.1 we know that $K_{d+1}(G)^{(d-1)} \leqq K_{1}(G), \cdots, K_{d+1}(G) \leqq K_{d}(G)$. Thus $K_{d+1}(G)=K_{d}(G)$ so that the upper $\mathscr{Q}$ series becomes stationary after at most $d$ steps, that is it has length at most $d$.

The Wielandt subgroup of a group $G$ is the intersection of the normalisers of all the subnormal subgroups of $G$, and will be denoted by $W(G)$. This subgroup was first introduced by Wielandt in [15]. We note that $W(G)$ is a characteristic subgroup of $G$ and that every subnormal subgroup of $W(G)$ is normal in $W(G)$ :

Definition. The upper Wielandt series of a group $G$ is defined as follows:

Let $W_{0}(G)=1$; if $\alpha$ is not a limit ordinal let $W_{\alpha}(G) / W_{\alpha-1}(G)=W\left(G / W_{\alpha-1}\right.$ $(G)$ ); if $\alpha$ is a limit ordinal let $W_{\alpha}(G)=\bigcup_{\beta<\alpha} W_{\beta}(G)$.

THEOREM 4.4. Let $G \in \mathrm{P} \mathscr{Q}$, and suppose that every subnormal subgroup of $G$ is normal. Then $G$ is abelian.

Proof. By a result of Robinson in [9] soluble groups in which normality is transitive are metabelian, so that $G \in \mathscr{Q}^{2}$. By $4.2 G^{\prime} \in \mathscr{Q}$. Let $A \leqq G^{\prime}$ with $A$ isomorphic to $C_{p} \infty$. A is subnormal in $G$ and so $A \triangleleft G$. Now $G / C_{G}(A)$ is isomorphic to a subgroup of the automorphism group of $A$. But $A$ satisfies the minimum condition and so by a well-known result its automorphism group is residually finite. Thus $G / C_{G}(A)$ is a residually finite 2 -group. But a residually finite radicable group is trivial, so that $A$ is contained in the centre of $G$. This implies that $G^{\prime}$ is contained in the centre of $G$, so that $G$ is nilpotent. A nilpotent periodic quasi-radicable group is abelian by a result of Černikov [2], and so $G$ is abelian.

We are now in a position to consider the lengths of the various series in $P \mathscr{Q}$ groups.

THEOREM 4.5. If $G \in \mathrm{P} \mathscr{Q}$ then the following invariants coincide: 
(i) the derived length $d(G)$ of $G$,

(ii) the length $q(G)$ of the upper 2 -series of $G$,

(iii) the nilpotent length $n(G)$ of $G$,

(iv) the Wielandt length $w(G)$ of $G$.

Proof. We prove first that $d(G)=q(G)$. By $4.3 q(G) \leqq d(G)$. On the other hand each factor of the upper $\mathscr{Q}$ series is abelian and so $d(G) \leqq q(G)$. Hence we have equality.

Next we show that $d(G)=n(G)$. Clearly $d(G) \geqq n(G)$. Let $1=G_{n} \triangleleft G_{n-1}$ $\triangleleft \cdots \triangleleft G_{1} \triangleleft G$ be a nilpotent series in $G$ of shortest length. We use induction on $d(G)$. Now $G / G_{1}$ is a nilpotent $\mathbf{P} \mathscr{2}$ group, so by Cernikov 's result again we have $G^{\prime} \leqq G_{1}$. Now $d(G)-1=$ derived length of $G^{\prime}$. But by the induction hypothesis $d\left(G^{\prime}\right) \leqq$ nilpotent length of $G^{\prime}$. Hence $d(G)-1 \leqq n\left(G^{\prime}\right) \leqq n\left(G_{1}\right)=$ $n(G)-1$. Thus $d(G) \leqq n(G)$ and so we have equality.

It only remains to show that $w(G)=d(G)$. Now $K_{1}(G)$ is a normal 2 subgroup of the periodic group $G$. Hence by $3.2 K_{1}(G) \leqq W_{1}(G)$. It is easy to see that by induction we have $K_{i}(G) \leqq W_{i}(G)$ for all $i$. Hence $w(G) \leqq q(G)$, and in particular $G$ has finite Wielandt length.

Suppose $A$ is a subnormal subgroup of a group $G$. We show by induction that $\quad W_{m}(A) \geqq A \cap W_{m}(G)$. By hypothesis $W_{m-1}(A) \geqq A \cap W_{m-1}(G)$. If $S / W_{m-1}(A)$ is a subnormal subgroup of $A / W_{m-1}(A)$ then $S$ is subnormal in $A$, and hence also in $G$. Hence $S W_{m-1}(G) / W_{m-1}(G)$ is subnormal in $G / W_{m-1}(G)$ and so $W_{m}(G)$ normalises $S W_{m-1}(G)$. Hence $W_{m}(G) \cap A$ normalises $S\left(W_{m-1}(G) \cap\right.$ $(A)=S$, and so $W_{m}(G) \cap A \leqq W_{m}(A)$.

Let us now return to the matter in hand; proving that $w(G) \geqq q(G)$. We have $W_{w}(G)=G$ where $w(q)=w, W_{w-1}(G)<G$. Now $G / W_{w-1}(G)=W\left(G / W_{w-1}(G)\right.$ and so is a $\mathrm{P} \mathscr{2}$ group in which normality is transitive. Then by theorem 4.4 $G^{\prime} \leqq W_{w-1}(G)$. Substituting $G^{\prime}$ for $A$ above we have $W_{w-1}\left(G^{\prime}\right) \geqq G^{\prime} \cap W_{w-1}$ $(G)=G^{\prime}$. Thus the Wielandt length of $G^{\prime}$ is at most $w-1$. If we again use induction on $d(G)=q(G), q(G)-1 \leqq w-1$ and so $q(G) \leqq w(G)$. Therefore we have equality, $q(G)=w(G)$.

We note that although these four series in a $\mathrm{P} \mathscr{Q}$ group turn out to have the same length there is no reason to suppose that they coincide term by term. In fact counterexamples are easy to construct: take $C_{t} \infty \times\left(C_{p} \infty w r C_{q} \infty\right)$, where this last group is the standard restricted wreath product of $C_{p} \infty$ by $C_{q} \infty$. The first term of the upper $\mathscr{Q}$-series of this group is $C_{t} \infty \times$ base group of the wreath product, whereas the derived group is the base group.

The next part of this section on $P \mathscr{Q}$ groups concerns their subnormal structure, and their effect on the subnormal structure of groups in which they occur as subnormal subgroups.

LEMMA 4.6. If $H$ is a periodic subnormal subgroup of an arbitrary group $G$ then $H K_{\alpha}(G)$ is normal in $H K_{\alpha+1}(G)$ for all $\alpha$. 
Proof. Since $H$ is subnormal in $G$ and $K_{\alpha}(G)$ is a normal subgroup, $H K_{\alpha}(G)$ is subnormal in $G$. Thus $H K_{\alpha}(G) / K_{\alpha}(G)$ is a periodic subnormal subgroup of $G / K_{\alpha}(G)$. But $K_{\alpha+1}(G) / K_{\alpha}(G)$ is a normal 2 subgroup of $G / K_{\alpha}(G)$ and so by 3.2 $K_{\alpha+1}(G) / K_{\alpha}(G)$ normalises $H K_{\alpha}(G) / K_{\alpha}(G)$. Hence $H K_{\alpha}(G) \triangleleft H K_{\alpha+1}(G)$.

THEOREM 4.7.

a) If $H$ is a subnormal subgroup of $G$, and $G \in \mathbf{P} \mathscr{Q}$ then $s(G: H) \leqq q(G)$. Thus $G$ has a bound on its subnormal indices.

b) If $H \in \mathbf{P} \mathscr{Q}$ and $H$ is subnormal in $G$ then $s(G: H) \leqq q(H)+1$.

c) $\mathrm{P} \mathscr{Q}$ is a subnormal coalition class. That is if $H$ and $K$ are subnormal $\mathrm{P} \mathscr{Z}$ subgroups of $G$ then their join $J=\langle H, K\rangle$ is a subnormal $\mathrm{P} \mathscr{Q}$ subgroup of $G$. Also we have $s(G: J) \leqq \max \{q(H), q(K)\}+1$.

Proof. a) This follows immediately from lemma 4.6 for we have $H \triangleleft$ $H K_{1}(G) \triangleleft H K_{2}(G) \triangleleft \cdots \triangleleft H K_{q}(G)=G$.

b) We show by induction that $K_{i}(G) \geqq K_{i}(H)$ for any subnormal subgroup $H$ of $G$. Suppose therefore that $K_{i-1}(G) \geqq K_{i-1}(H)$. Suppose that $S / K_{i-1}(H)$ is a subnormal 2 subgroup of $H / K_{i-1}(H)$. Then $S$ is subnormal in $H$, and so also in $G$. Therefore $S K_{i-1}(G) / K_{i-1}(G)$ is a subnormal subgroup of $G / K_{i-1}(G)$. $S K_{i-1}(G) / K_{i-1}(G) \cong S / S \cap K_{i-1}(G)$, which is a homomorphic image of $S / K_{i-1}(H)$ since $K_{i-1}(G) \geqq K_{i-1}(H)$ by hypothesis. Thus $S K_{i-1}(G) / K_{i-1}(G)$ is a $\mathscr{Q}$ group. Thus $S K_{i-1}(G) \leqq K_{i}(G)$. It follows from the choice of $S$ that $K_{i}(H) \leqq K_{i}(G)$. Thus if $H$ is subnormal in $G$ we have $K_{i}(H) \leqq K_{i}(G)$ for all $i$.

Now let $H \in \mathrm{p} \mathscr{Q}$ with $q=q(H)$. Then we have $K_{q}(G) \geqq K_{q}(H)=H$. Thus $H$ is a subnormal subgroup of $K_{q}(G)$, and so by a) $H \triangleleft^{q} K_{q}(G) \triangleleft G$. Therefore $s(G: H) \leqq q(H)+1$ as required.

c) Let $H, K$ be subnormal $\mathbf{P} \mathscr{Q}$ subgroups of $G$. Let $n=\max \{q(H), q(K)\}$. Then by the argument used in b) $K_{n}(H) \leqq K_{n}(G), K_{n}(K) \leqq K_{n}(G)$. But $H=K_{n}(H)$ and $K=K_{n}(K)$. Thus $J=\langle H, K\rangle \leqq K_{n}(G)$. But by a) $K_{n}(G)$ is a group with a bound for its subnormal indices. Then it is easy to show that the join of an arbitrary collection of subnormal subgroups of $K_{n}(G)$ is subnormal in $K_{n}(G)$ (see, for instance p 85 of [13]). Thus $J$ is subnormal in $G$. It remains to show that $J \in \mathbf{P} \mathscr{2}$. Again as in b) we have $K_{n}(H) \leqq K_{n}(J)$ and $K_{n}(K) \leqq K_{n}(J)$, so that $J \leqq K_{n}(J)$. Therefore $J=K_{n}(J)$ and $J$ is a $\mathrm{P} \mathscr{Q}$ group.

We now turn to the locally nilpotent structure of $\mathrm{p} \mathscr{Q}$ and $\dot{P} \mathscr{Q}$ groups.

\section{THEOREM 4.8.}

(i) The lower central series of a $\mathbf{P} \mathscr{Q}$ group becomes stationary at the derived group. Thus $G^{\prime}=\left[G^{\prime}, G\right]=\cdots$.

(ii) The Baer radical of a $\mathbf{P} 2$ group of length $n$ is nilpotent of class at most $n$.

(iii) If $G$ is a $\mathbf{P} \mathscr{Q}$ group and is also a Baer group then it is abelian.

Proof. (i) Let $G \in \dot{\mathbf{P}} \mathscr{Q}$. Then $G /\left[G^{\prime}, G\right]$ is a nilpotent periodic group and is 
quasi-radicable. Hence using Cernikov's result again $G /\left(G^{\prime}, G\right]$ is abelian. Thus $G^{\prime} \leqq\left[G^{\prime}, G\right] \leqq G^{\prime}$. Therefore $G^{\prime}=\left[G^{\prime}, G\right]=\left[G^{\prime}, G, G\right]$.

(ii) Suppose $G \in \mathbf{P} \mathscr{2}$ with $q(G)=n$. We proceed by induction on $n$. Now $G^{(n-1)} \in \mathscr{Q}$ and so by lemma 3.2 it commutes with every subnormal nilpotent subgroup of $G$. Hence $G^{(n-1)}$ is contained in the centre of $B$, where $B$ is the Baer radical of $G$. However $B / G^{(n-1)}$ is contained in the Baer radical of $G / G^{(n-1)}$, which by the induction hypothesis is nilpotent of class at most $n-1$. Thus $B$ is nilpotent of class at most $n$.

(iii) If $G \in \mathbf{P} \mathscr{Q}$ and is a Baer group then $K_{1}(G)$ is in the centre of $G$ by lemma 3.2. By transfinite induction it clearly follows that $G$ is a $Z A$ group. But $G$ is periodic and quasi-radicable, so by Černikov's result $G$ is abelian.

In [8] McLain shows that the group $C_{p} \infty w r C_{q} \infty$ coincides with its Frattini subgroup. However the proof he gives can be adapted to give the following more general result.

THEOREM 4.9. If $G \in \dot{P} \mathscr{Q}$ then $G$ has no maximal subgroups and so coincides with its Frattini subgroup.

Proof. By lemma $4.1 G \in \dot{\mathrm{P}}_{\mathrm{N}} \mathscr{Q}$ so that $G=K_{\rho}(G)$ for some ordinal $\rho$. Suppose that $M$ is a proper maximal subgroup of $G$. Let $r$ be the smallest ordinal such that $K_{r}(G) \$ M$. Clearly $r$ cannot be a limit ordinal so that $r-1$ exists. Now $K_{r-1}(G) \leqq M$ and $M / K_{r-1}(G)$ is a maximal subgroup of $G / K_{r-1}(G)$, which belongs to $\dot{\mathbf{P}} \mathscr{Q}$, and so we may assume $K_{r-1}(G)=1$. Let $A=K_{r}(G) \in \mathscr{Q}$. In Lemma 10 of [4] P. Hall shows that if $G$ is any group and $A$ is a normal abelian $p$-subgroup of $G$ where $p$ is a prime, then the Frattini subgroup of $G$ contains $A^{p}$. Using this lemma we see that in our case $M \geqq A^{p}$ for all primes $p$. But $A=A^{p}$, which gives a contradiction. Hence $G$ has no maximal subgroups. We are grateful to the referee for pointing out this simplification of our original proof.

\section{Soluble $p$-groups}

Definitions. A group $G$ is reduced if it has no non-trivial quasi-radicable subgroups. If $\pi$ is any set of primes a group $G$ is $\pi$-quasi-radicable if $G=G^{m}$ for all positive integers $m$ whose prime divisors lie in $\pi$. A group $G$ is $\pi$-reduced it has no non-trivial $\pi$-quasi-radicable subgroups.

Lemma 5.1. Let $G=\langle x, A\rangle$ be a p-group with $A$ abelian and normal in $G$. If $A$ is reduced and $G$ has a bound on its subnormal indices then $G$ is nilpotent.

Proof. We define a descending series of characteristic subgroups of $A$ as follows:

Let $A_{0}=A$; if $\alpha$ is not a limit ordinal let $A_{\alpha}=A_{\alpha-1}{ }^{p}$; if $\alpha$ is a limit ordinal let $A_{\alpha}=\bigcap_{\beta<\alpha} A_{\beta}$. Then for some ordinal $\lambda, A_{\lambda}=A_{\lambda+1}=\cdots=D$. Now 
$D=D^{p}$ so that $D$ is radicable (remembering that $D$ is a $p$-group). But $D$ is contained in $A$ and by assumption $A$ is reduced. Hence $D=1$. Suppose $G$ is not nilpotent, and let $\alpha$ be the least ordinal such that $G / A_{\alpha}$ is not nilpotent. Now if $\alpha$ is a limit ordinal $G / A_{\beta}$ is nilpotent for all $\beta<\alpha$. Roseblade ([14], corollary to Theorem 1) has shown that a group in which every subgroup is subnormal with subnormal index at most $r$, is nilpotent of class at most $f(r)$ depending only on $r$. If $r$ is the bound on the subnormal indices of $G$ then each $G / A_{\beta}, \beta<\alpha$, has the same bound. Hence each is nilpotent of class at most $f(r)$. Then $G / A_{\alpha}$ is nilpotent of class at most $f(r)$, which is a contradiction. Thus $\alpha$ has a predecessor $\alpha-1 . G / A_{\alpha-1}$ is nilpotent and $x$ has order $p^{m}$ for some positive integer $m$. Let $a \in A_{\alpha-1}$. Then we have

that is

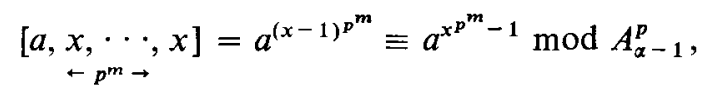

$$
\left[a, \underset{-p^{m} \rightarrow}{x, \cdots}\right] \equiv 1 \bmod A_{\alpha} .
$$

Hence $\gamma A_{\alpha-1}\langle x\rangle^{p^{m}} \leqq A_{\alpha}$ and thus $\gamma A_{\alpha-1} G^{p^{m}} \leqq A_{\alpha}$. Therefore $G / A_{\alpha}$ is nilpotent, which is a contradiction. Hence $G$ is nilpotent.

LEMMA 5.2. Let $G$ be a metabelian p-group in which there is a bound on the subnormal indices. If the abelian normal subgroups of $G$ are reduced then $G$ is nilpotent.

Proof. Let $A=G^{\prime}$. Then by hypothesis $A$ is reduced. Let $g \in G$. Then $\langle g, A\rangle$ is normal in $G$ and so $\langle g, A\rangle$ has a bound on its subnormal indices. So by lemma $5.1\langle g, A\rangle$ is nilpotent. Therefore $\langle g\rangle$ is subnormal in $\langle g, A\rangle$, and hence also in $G$. It is easy to show that since $G$ has a bound on its subnormal indices the join of any collection of subnormal subgroups of $G$ is subnormal (p. 85 of [13]). Hence every subgroup of $G$ is subnormal. Then using Roseblade's result we see that $G$ is nilpotent.

We would like to extend this result to a soluble p-group of arbitrary derived length. To do so we need the following lemma, which is stated in a more general form than is strictly necessary.

Lemma 5.3. Let $A$ and $B$ be abelian groups and let $H=\operatorname{Hom}(A, B)$. If $\pi$ is any set of primes and $B$ is $\pi$-reduced then $H$ is $\pi$-reduced.

Proof. Suppose on the contrary that $R$ is a $\pi$-quasi-radicable subgroup of $H$. Let $a \in A$. Then the mapping $\theta: r \rightarrow a r$ for $r \in R$ is a homomorphism of $R$ into $B$. Hence $\operatorname{Im} \theta$ is a $\pi$-quasi-radicable subgroup of $B$. Since $B$ is $\pi$-reduced $\theta$ is the zero-mapping, so that $a r=1$ for all $a \in A$ and $r \in R$. Hence $R$ is trivial.

Theorem 5.4. Let $G$ be a soluble p-group with a bound for its subnormal indices. If every abelian normal subgroup of $G$ is reduced then $G$ is nilpotent. 
Proof. We may assume that the derived length of $G$ is greater than 1 . We use induction on $d$, the derived length of $G$.

Let $A$ be a maximal abelian normal subgroup of $G$ containing $G^{(d-1)}$. We must show that $G / A$ has the right properties to allow us to apply the induction hypothesis. Suppose $R / A$ is a radicable abelian normal subgroup of $G / A$. If we can show that $R$ is abelian then $R=A$ by the maximality of $A$. Let $x \in R$. Then $\langle x, A\rangle \triangleleft R \triangleleft G$ and so $\langle x, A\rangle$ has a bound on its subnormal indices. A is reduced and so by lemma $5.1\langle x, A\rangle$ is nilpotent. Thus $\langle x\rangle$ is subnormal in $R$ for all $x \in R$. Since $R$ has a bound on its subnormal indices every subgroup of $R$ is therefore subnormal in $R$. Hence by Roseblade's result $R$ is nilpotent. Therefore $\gamma A R^{n}=1$ for some integer $n$. Choose $n$ minimal with respect to this property. Suppose $n>1$. Then $\gamma A R^{n-2}>\gamma A R^{n-1}>\gamma A R^{n}=1$. For $x \in R$ and $a \in \gamma A R^{n-2}$ we define $\tau_{x}: a \gamma A R^{n-1} \rightarrow[a, x]$. Then $\tau_{x}$ is a homomorphism of $\gamma A R^{n-2} / \gamma A R^{n-1}$ into $\gamma A R^{n-1}$. The mapping $\tau: x \rightarrow \tau_{x}$ is a homomorphism of $R$ into Hom $\left(\gamma A R^{n-2} /\right.$ $\left.\gamma A R^{n-1}, \gamma A R^{n-1}\right)=H$ say. Applying lemma 5.3 we have that $H$ is reduced. Now $R / \operatorname{Ker} \tau$ is isomorphic to a subgroup of $H$, and so is reduced. However $\operatorname{Ker} \tau$ is the centralizer in $R$ of $\gamma A R^{n-2}$ and so contains $A$. Thus $R / \operatorname{Ker} \tau$ is radicable. Therefore $R=\operatorname{Ker} \tau$ and so $\gamma A R^{n-1}=1$, which is a contradiction. Thus $n=1$ and $\gamma A R=1$, so that $A \leqq$ centre of $R$. Let $r \in R$. Then the mapping $x A \rightarrow[x, r]$ for all $x \in R$ is a homomorphic mapping of $R / A$ onto $[R, r] . R / A$ is radicable and so $[R, r]$ is radicable. However $[R, r] \leqq A$ since $R / A$ is abelian, and $A$ is reduced. Thus $[R, r]=1$ for all $r \in R$. Hence $R$ is abelian. By the maximality of $A$ we have $R=A$, and so the abelian normal subgroups of $G / A$ are reduced. Hence by the induction hypothesis $G / A$ is nilpotent.

Let $x \in G$. Since $G / A$ is nilpotent $\langle x, A\rangle$ is subnormal in $G$ and so $\langle x, A\rangle$ has a bound on its subnormal indices. By lemma $5.1\langle x, A\rangle$ is nilpotent. Thus $\langle x\rangle$ is subnormal in $G$. Since $G$ has a bound on its subnormal indices this implies that every subgroup of $G$ is subnormal, and so by Roseblade's result $G$ is nilpotent.

We can now prove our result concerning soluble $p$-groups with a bound on their subnormal indices.

THEOREM 5.5. Let $G$ be a soluble p-group. Then $G$ has a bound on its subnormal indices if and only if $G$ is an extension of a $\mathbf{P} \mathscr{Q}$ group by a nilpotent group.

Proof. a) Suppose $G$ has a bound on its subnormal indices. Let $n$ be the derived length of $G$. By lemma $4.3 K_{n}(G)=K_{n+1}(G)=\cdots$ and $K_{n}(G) \in \mathbf{P} \mathscr{Q}$. Consider $G / K_{n}(G)$. By the definition of the upper $\mathscr{Q}$-series any radicable abelian normal subgroup of $G / K_{n}(G)$ is contained in $K_{n+1}(G) / K_{n}(G)=1$. Thus $G / K_{n}(G)$ satisfies the conditions of theorem 5.4. Hence $G / K_{n}(G)$ is nilpotent.

b) Suppose conversely that $N \triangleleft G$ with $G / N$ nilpotent and $N \in \mathrm{P} \mathscr{Q}$. Suppose $G / N$ has class $m$ and $N$ has length $n$. Then if $H$ is a subnormal subgroup of $G$ we have $H N \triangleleft^{m} G$. By lemma $4.6 H \triangleleft^{n} H K_{n}(H N)$. But we also have 
$K_{n}(N) \leqq K_{n}(H N)$, and $K_{n}(N)=N$. Thus $H \triangleleft^{n} H N \triangleleft^{m} G$. Therefore $s(G: H)$ is at most $m+n$, and so $G$ has a bound on its subnormal indices.

COROLlary 5.6. Let $G$ be a soluble locally nilpotent periodic group. Then $G$ has a bound on its subnormal indices if and only if $G$ is an extension of a $\mathrm{P} \mathscr{Q}$ group by a nilpotent group.

Proof. Suppose $G$ has derived length $d$ and has a bound $n$ on its subnormal indices. Since $G$ is periodic and locally nilpotent it is a direct product of its $p$ primary subgroups $G_{p}$. Each $G_{p}$ is a soluble $p$-group with a bound on its subnormal indices. So by theorem 5.5 and Roseblade's result $G_{p}$ is the extension of a $\mathscr{Q}^{d}$ group (a $\mathrm{P} \mathscr{Q}$ group of derived length $d$ ) by a nilpotent group of class at most $f(n)$, for all $p$. Hence $G$ is of the same form. The reverse implication is immediate from the proof of 5.5 .

\section{Application to locally nilpotent groups}

In a nilpotent group $G$ of class $n$ the subnormal subgroups have subnormal index at most $n$. It is natural therefore to look at locally nilpotent groups with a bound on their subnormal indices. However this class seems to be too large. But we can show that periodic $Z A$ groups with a bound on their subnormal indices are soluble. Perhaps all periodic locally nilpotent groups with a bound on their subnormal indices are soluble, but at the moment it remains an open question.

LemMa 6.1. A Baer group with a bound on its subnormal indices is nilpotent.

Proof. Since $G$ is a Baer group $\langle x\rangle$ is subnormal in $G$ for all elements $x$ of $G$. But since $G$ has a bound on its subnormal indices every subgroup of $G$ is a subnormal subgroup. Then by Roseblade's result $G$ is nilpotent.

THEOREM 6.2. Let $G$ be a periodic $Z A$ group. Then $G$ has a bound on its subnormal indices if and only if $G$ is an extension of a 2 group by a nilpotent group.

Proof. If $G$ is an extension of a $\mathscr{Q}$ group by a nilpotent group then we see from the proof of 5.5 that $G$ has a bound on its subnormal indices.

Suppose, conversely, that $G$ has a bound on its subnormal indices. Since $G$ is the direct product of its $p$-components we may assume that $G$ is a $p$-group (using Roseblade's result). Let $R$ be the subgroup of $G$ generated by all the quasi-radicable subgroups of $G$. Clearly $R$ is the maximal quasi-radicable subgroup of $G$ and is normal in $G$. But by Cernikov's result a periodic quasi-radicable $Z A$ group is abelian, and so is a $\mathscr{Q}$ group. Thus it remains to show that $G / R$ is nilpotent. Clearly $G / R$ has no radicable abelian subgroups. Let $\left\{Z_{\alpha}\right\}_{\alpha \leqq \rho}$ be the upper central series of $G / R$. Suppose $G / R$ is not nilpotent. Let $\lambda$ be the least ordinal such that $Z_{\lambda}$ is not nilpotent. If $\lambda$ is a limit ordinal $Z_{\lambda}=\bigcup_{\mu<\lambda} Z_{\mu}$ where each $Z_{\mu}$ is nil- 
potent of class at most $f(r)$, using Roseblade's result with the subnormal indices of $G$ bounded by $r$. Hence $Z_{\lambda}$ is nilpotent, which is a contradiction. Thus $\lambda-1$ exists and $Z_{\lambda-1}$ is nilpotent. Then $Z_{\lambda}$ is a soluble $p$-group satisfying the hypotheses of 5.4 , and so is nilpotent, which is a contradiction. Therefore $G / R$ is nilpotent, as required.

Finally we have the following results on $S I^{*}$ groups.

THEOREM 6.3. Let $G$ be a p-group and also an $S I^{*}$ group. Suppose that all the subnormal abelian subgroups of $G$ are reduced. If $G$ has a bound on its subnormal indices then it is nilpotent.

Proof. Let $\left\{G_{i}\right\}_{i \leqq \rho}$ be an ascending normal abelian series of $G$ with $G_{\rho}=G$. Suppose $G$ is not nilpotent. Let $\lambda$ be the least ordinal such that $G_{\lambda}$ is not nilpotent. If $r$ is the bound on the subnormal indices, and if $\lambda$ is a limit ordinal, then $G_{\lambda}=$ $\bigcup_{\mu<\lambda} G_{\mu}$ where each $G_{\mu}$ is nilpotent of class at most $f(r)$. Hence $G_{\lambda}$ is nilpotent, which is a contradiction. Thus $G_{\lambda-1}$ is nilpotent, and so $G_{\lambda}$ is a soluble $p$-group with all its abelian normal subgroups reduced. Therefore by theorem $5.4 G_{\lambda}$ is nilpotent, which is a contradiction. Hence $G$ is nilpotent.

COROLLARY 6.4. If $G$ is an SI* group, a p-group, and has a bound on its subnormal indices then it is an extension of a $\mathbf{P} 2$ group by a nilpotent group.

Proof. Let $K_{\lambda}(G)$ be the last term of the upper $\mathscr{Q}$ series of $G$, so that $K_{\lambda}(G)=$ $K_{\lambda+1}(G)$. Then $K_{\lambda}(G) \in \dot{\mathbf{P}} \mathscr{Q}$ and $G / K_{\lambda}(G)$ satisfies the conditions of theorem 6.3. Hence the result follows.

The corollary gives rise to the open question: is there a $\mathbf{P} \mathscr{2}$ group which is a $p$-group, has a bound on its subnormal indices, but is not soluble? If so the structure of periodic locally nilpotent groups with a bound on their subnormal indices is very complex.

\section{Acknowledgement}

The material in this paper forms part of a Ph. D. thesis for the University of London. I wish to thank my supervisor Dr D. J. S. Robinson for his valuable help and encouragement, and the Science Research Council for their financial support.

\section{References}

[1] E. Best and O. Taussky, 'A class of groups', Proc. Roy. Irish Acad. Sect. A 47 (1942), 55-62.

[2] S. N. Cernikov, 'Complete groups with an ascending central series', Mat. Sbornik 18 (1946), 397-422.

[3] W. Gaschütz, 'Gruppen, in denen das Normalteilersein transitiv ist', J. rein u. angew. Math. 198 (1957), 87-92. 
[4] P. Hall, 'The Frattini subgroups of finitely generated groups', Proc. London Math. Soc. (3) $11(1961), 327-352$.

[5] P. Hall, 'On non-strictly simple groups', Proc. Camb. Phil. Soc. 59 (1963), 531-553.

[6] M. Krasner et L. Kaloujnine, 'Produit complet des groupes de permutations et le problème d'extension des groupes. III', Acta. Sci. Math. Szeged 14 (1951), 69-82.

[7] A. G. Kuroš, The theory of groups (2nd edition, New York, 1956).

[8] D. H. McLain, 'On locally nilpotent groups', Proc. Camb. Phil. Soc. 52 (1956), 5-11.

[9] D. J. S. Robinson, 'On groups in which normality is transitive', Proc. Camb. Phil. Soc. 60 (1964), $21-38$.

[10] D. J. S. Robinson, 'On finitely generated soluble groups', Proc. London Math. Soc. (3) 15 (1965), 508-516.

[11] D. J. S. Robinson, 'On the theory of subnormal subgroups', Math. Zeit. 89 (1965), 30-51.

[12] D. J. S. Robinson, 'Wreath products and indices of subnormality', Proc. London Math. Soc. (3) 17 (1967), 257-270.

[13] D. J. S. Robinson, Infinite soluble and nilpotent groups (Queen Mary College, London, 1968).

[14] J. E. Roseblade, 'On groups in which every subgroup is subnormal', J. of Algebra 2 (1965), 402-412.

[15] H. Wielandt, 'Über den Normalisator der subnormalen Untergruppen', Math. Zeit. 69 (1958), 463-465.

[16] G. Zacher, 'Caratterizzazione dei $t$-gruppi finiti risolubili', Ricerche Mat. 1 (1952), 287-294.

Institute of Advanced Studies

Australian National University

Canberra A.C.T. 2600 